\title{
Caracterización del dolor en las pacientes con cáncer de mama que asisten a la Unidad de Oncología del Hospital San José
}

\author{
C. L. Buitrago Martín¹, J. Orlando Pacheco², D. Carolina Estrada ${ }^{3}$ y C. Mario Acevedo ${ }^{4}$
}

${ }^{1}$ Instructora asociada, Departamento de Dolor y Cuidado Paliativo, Fundación Universitaria de Ciencias de la Salud, Hospital de San José, Bogotá, Colombia. ${ }^{2}$ Instructor asistente, Departamento de Oncología, Fundación Universitaria de Ciencias de la Salud, Hospital de San José, Bogotá, Colombia. ${ }^{3} F e l l o w ~ s e g u n d o$ año Dolor y Cuidado Paliativo. Fundación Universitaria de Ciencias de la Salud, Hospital de San José, Bogotá, Colombia. ${ }^{4}$ Fellow segundo año Dolor y Cuidado Paliativo, Fundación Universitaria de Ciencias de la Salud, Hospital de San José, Bogotá, Colombia

\section{ABSTRACT}

Introduction: Breast cancer ranks second in the world and is the most frequent in women with 1.67 million new cases In 2012, it was 25\% of all cancers, it is projected for 2030 more than 596,000 new cases and an increase of 142,100 deaths from breast cancer. One of the most frequent symptoms experienced by these patients is acute or chronic pain, which can occur at any time of the disease (Diagnosis, treatment, remission or relapse), should be evaluated and diagnosed, avoiding chronicity as much as possible. confers a degree of greater difficulty for its control.

Objectives: To determine the presence of pain in breast cancer patients undergoing cancer treatment (chemotherapy, radiotherapy, surgery, and / or hormonal treatment), and to describe the characteristics thereof.

Methods: Descriptive cross-sectional study in patients attending the oncology service of San José Hospital with a diagnosis of breast cancer and who are undergoing oncological treatment through a personal survey.

Results: Data were collected from 93 patients, of which $56.9 \%$ presented pain, with a higher prevalence of chronic pain in $52.8 \%$; $54.7 \%$ classified their pain as nociceptive and $45.2 \%$ as neuropathic pain with intense pain in $49.0 \%$. The pain was treated in $52.8 \%$ with acetaminophen, and $22.6 \%$ had no treatment.

Key words: Cancer pain, breast cancer, oncological treatment, neuropathy.

\section{RESUMEN}

Introducción: El cáncer de mama ocupa el segundo lugar en el mundo y es el más frecuente en mujeres con presencia de 1,67 millones de casos nuevos. En 2012 supuso el $25 \%$ de todos los cánceres, y se proyectan para 2030 más de 596.000 nuevos casos y un aumento de 142.100 muertes por cáncer de mama. Uno de los síntomas más frecuentes que experimentan estas pacientes es el dolor agudo o crónico, el cual puede ocurrir en cualquier momento de la enfermedad (diagnóstico, tratamiento, remisión o recaída); por esto debe evaluarse y diagnosticarse, evitando la cronicidad tanto como sea posible. En Colombia no existen estudios referentes al tema.

Objetivos: Determinar la presencia de dolor en pacientes con cáncer de mama sometidas a tratamiento oncológico (quimioterapia, radioterapia, cirugía y/o tratamiento hormonal] y describir sus características.

Métodos: Estudio observacional descriptivo de corte transversal en pacientes atendidas en el servicio de oncología de un hospital de cuarto nivel en Bogotá (Colombia), con diagnóstico de cáncer de mama y en tratamiento oncológico. Las variables cualitativas fueron descritas con frecuencias absolutas y relativas y las cuantitativas con medias.

Resultados: Se recolectaron los datos de 93 pacientes, de las que el 56,9\% presentaron dolor, con mayor prevalencia de dolor crónico en un 52,8\%; el 54,7\% clasificaron su dolor como nociceptivo y el $45,2 \%$ como dolor neuropático con dolor intenso en el 49,0 \%. El dolor fue tratado en el 52,8 \% con acetaminofeno, y un 22,6\% no tenían ningún tratamiento.

Palabras clave: Dolor oncológico, cáncer de mama, tratamiento oncológico, neuropatía.

Recibido: 31-05-2018

Aceptado: 26-03-2019

Buitrago Martín CL, Orlando Pacheco J, Carolina Estrada D, Mario Acevedo C. Caracterización del dolor en las pacientes con cáncer de mama que asisten a la unidad de oncología del Hospital San José. Rev Soc Esp Dolor 2019;26(4):221-226.
Correspondencia: Claudia Liliana Buitrago Martín claudiabuitrago.doc@gmail.com 
Rev. Soc. Esp. del Dolor, Vol. 26, N. ${ }^{\circ} 4$, Julio-Agosto 2019

\section{INTRODUCCIÓN}

El cáncer de mama ocupa el segundo lugar en el mundo y es el más frecuente en mujeres, con la presencia de 1,67 millones de casos nuevos. En 2012 supuso el $25 \%$ de todos los cánceres, y se proyectan para el año 2030 más de 596.000 nuevos casos y un aumento de 142.100 muertes por cáncer de mama (1). En Colombia, en el año 2013, el Instituto Nacional de Cancerología publicó una actualización donde reportó que se diagnostican 7000 casos nuevos de cáncer de mama cada año, y 2500 mujeres mueren por esta causa [2].

El tratamiento del cáncer de mama ha presentado avances gigantescos con el ingreso de la terapia genética, con altas tasas de curación y tiempos de supervivencia más altos. La base de datos National Cáncer Institute SEER, que incluye personas con diagnóstico de cáncer de seno entre 2007 y 2013, informa de tasa relativas de supervivencia que van desde el $100 \%$ en los estadio I al $72 \%$ en estadio III (3). No obstante, estos tratamientos y el cáncer en sí pueden generar el desarrollo de múltiples síntomas físicos, psicológicos y sociales, relacionados con la terapia oncológica. Entre ellos, el más frecuente es el dolor, que puede ocurrir en cualquier momento de la enfermedad (diagnóstico, tratamiento, remisión o recaída), y entre los relacionados con el tratamiento oncológico se incluye el dolor posquirúrgico persistente, la neuropatía periférica inducida por quimioterapia, el dolor asociado a radiotoxicidad, además del dolor articular inducido por los inhibidores de la aromatasa $[4,5]$.

En 1979, la Asociación Internacional para el Estudio del Dolor [IASP, por sus siglas en inglés] definió el dolor como "experiencia sensorial y emocional desagradable, asociada a una lesión tisular real o potencial descrita en términos de la misma" $(6,7)$. El enfermo con cáncer, al presentar varios factores de riesgo que le hacen susceptible para padecer afectación del nervio periférico y dolor nociceptivo, con varios factores que pueden llevar al desarrollo de dolor crónico (8), debe tener una evaluación adecuada del dolor para así establecer un diagnóstico y manejo oportunos, disminuyendo la presencia de síndromes dolorosos de difícil resolución que deterioren su calidad de vida (9).

El tratamiento del dolor oncológico debe centrarse en el paciente, y requiere un manejo multidisciplinario incluyendo salud mental, rehabilitación, clínica de dolor y cuidados paliativos, entre otros. Si la enfermedad progresa, el dolor experimentado aumenta en la EVA (escala visual analógica), lo que hace que se requiera la administración de opioides potentes y un manejo multimodal para lograr un adecuado control $[2,6]$.

Conociendo esto, sabemos que el dolor puede estar presente en cualquier fase de la enfermedad o del tratamiento, por lo cual debe ser valorado adecuadamente y así poder establecer un tratamiento oportuno. En Colombia no existen estudios que describan el manejo del dolor en las pacientes con cáncer de mama que están en tratamiento oncológico; por ello, consideramos importante conocer la prevalencia, las características del dolor y el manejo analgésico en las pacientes con diagnóstico de cáncer de mama que se encuentran en tratamiento oncológico en un centro hospitalario de cuarto nivel.

\section{MÉTODOS}

\section{Diseño}

Estudio observacional descriptivo de corte transversal.

\section{Población}

Se incluyeron pacientes mayores de 18 años con diagnóstico de cáncer de mama en tratamiento oncológico, valoradas ambulatoriamente en la Unidad de Oncología del Hospital de San José. Se excluyeron las pacientes con alteraciones en el estado de alerta o que se encontraran hospitalizadas.

Para el cálculo del tamaño de muestra se consideró una frecuencia de dolor esperada del $40 \%$ (5), un nivel de confianza del $95 \%$ y una precisión del $10 \%$, para un tamaño de muestra de 93 pacientes. El cálculo se realizó con el programa estadístico EPIDAT 4.1.

\section{Procedimiento}

A las pacientes dispuestas a participar en el estudio se les aplicó una encuesta verbal realizada durante su visita ambulatoria de oncología o durante la quimioterapia, además de tomar datos de la historia clínica, y se registraron variables sociodemográficas y variables clínicas: estadio de cáncer, tiempo de diagnóstico, tratamiento oncológico recibido y comorbilidades. Se interrogó por la presencia de dolor, características e intensidad del mismo, usando para ello la EVA; y para la presencia de dolor neuropático se usó la escala DN4 modificada de siete ítems (10), además del manejo farmacológico recibido para el dolor. Para el análisis de la variable intensidad del dolor, esta fue categorizada como:

- Dolor leve: EVA 1 a 3.

- Dolor moderado: EVA 4 a 6.

- Dolor intenso: EVA mayor o igual a 7.

El estudio fue aprobado por el comité de ética institucional.

\section{Análisis estadístico}

Las variables cualitativas fueron descritas por frecuencias absolutas y relativas. Las variables cuantitativas fueron descritas con medidas de tendencia central y dispersión: promedio y desviación estándar (DE) o mediana y rango intercuartílico, de acuerdo a su distribución, utilizando el paquete estadístico Stata 13. Las gráficas se realizaron en Excel.

\section{RESULTADOS}

Se incluyeron en el estudio 93 pacientes que cumplieron con los criterios de elegibilidad, las cuales se encontraban en rangos de edad de entre 30 y 90 años, con una media de 58 años (DE 12,4); en su mayoría tenían estudios de secundaria [44\%] y el 27,9\% eran solteras. 
De las comorbilidades, la más frecuente fue la hipertensión arterial, que padecían el 26,8 \% de las pacientes, seguida de la diabetes tipo 2 y el hipotiroidismo, que sufrían el $13,9 \%$ y el 10,7\%, respectivamente. El estadio inicial oncológico mostró que un 25,8 \% estaban en estadio IIA y un 21,5\% en estadio IIIA (Tabla I).

\section{Tratamiento oncológico}

La mayoría de las pacientes [56,9 \%) habían sido diagnosticadas de cáncer hace 5 años o menos, y su tratamiento oncológico se había iniciado durante este

TABLA I

CARACTERÍSTICAS GENERALES DE LAS PACIENTES DEL ESTUDIO

\begin{tabular}{|c|c|c|}
\hline & Número & Porcentaje \\
\hline \multicolumn{3}{|l|}{ Nivel de estudios } \\
\hline Analfabeta & 1 & $1,0 \%$ \\
\hline Primaria & 19 & $20,4 \%$ \\
\hline Secundaria & 41 & $44,0 \%$ \\
\hline Técnica & 16 & $17,2 \%$ \\
\hline Universitaria & 13 & $13,9 \%$ \\
\hline \multicolumn{3}{|l|}{ Estado civil } \\
\hline Casada & 28 & $30,1 \%$ \\
\hline Soltera & 26 & $27,9 \%$ \\
\hline Unión libre & 15 & $16,9 \%$ \\
\hline Viuda & 13 & $13,9 \%$ \\
\hline Separada & 11 & $11,8 \%$ \\
\hline \multicolumn{3}{|l|}{ Antecedentes médicos } \\
\hline Diabetes & 13 & $13,9 \%$ \\
\hline Hipertensión arterial & 25 & $26,8 \%$ \\
\hline Hipotiroidismo & 10 & $10,7 \%$ \\
\hline Tabaquismo & 3 & $3,2 \%$ \\
\hline Osteoporosis & 2 & $2,1 \%$ \\
\hline Ansiedad & 2 & $2,1 \%$ \\
\hline \multicolumn{3}{|l|}{ Estadificación tumoral } \\
\hline Desconocido & 3 & $3,23 \%$ \\
\hline IA & 8 & $8,6 \%$ \\
\hline $\mathrm{IB}$ & 1 & $1,0 \%$ \\
\hline$\| A$ & 24 & $25,8 \%$ \\
\hline IIB & 15 & $16,1 \%$ \\
\hline IIIA & 20 & $21,5 \%$ \\
\hline IIIB & 6 & $6,4 \%$ \\
\hline $\mathrm{IIIC}$ & 3 & $3,2 \%$ \\
\hline IV & 13 & $13,9 \%$ \\
\hline
\end{tabular}

Fuente: Autores. mismo periodo. Un 91,4\% habían recibido quimioterapia en algún momento de su tratamiento (85 pacientes) y el $76,3 \%$ habían sido intervenidas quirúrgicamente (Figura 1 y Tabla II).

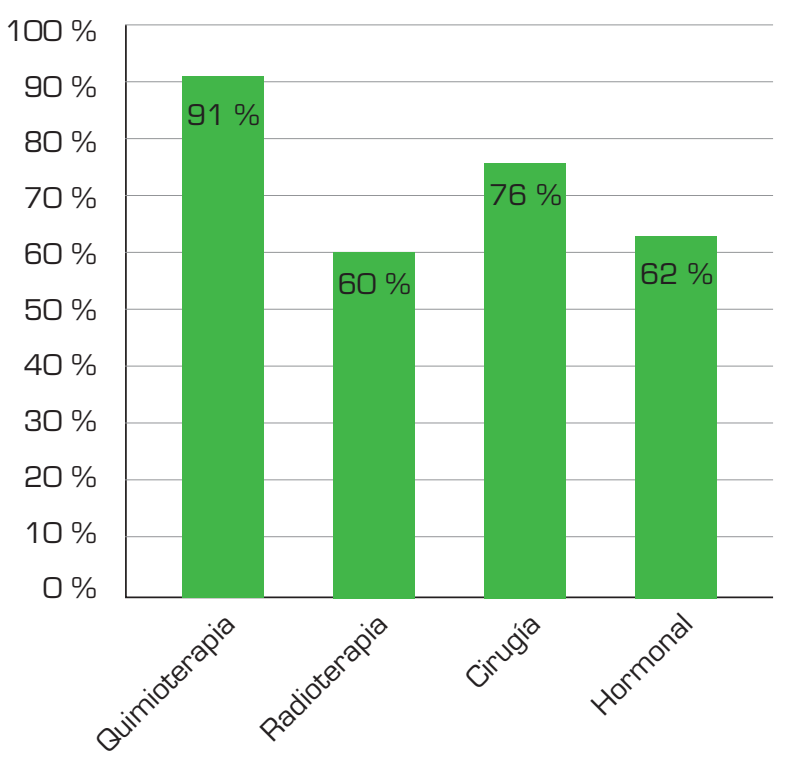

Fig. 1. Distribución del tipo de tratamiento recibido por las pacientes.

TABLA II

TRATAMIENTO ONCOLÓGICO

\begin{tabular}{|l|l|}
\hline \multicolumn{2}{|c|}{ Esquema de quimioterapia } \\
\hline Antracíclicos & $24,7 \%$ \\
\hline Antracíclicos + taxanos & $55,2 \%$ \\
\hline Antracíclicos + taxanos + trastuzumab & $7,0 \%$ \\
\hline Taxanos & $4,7 \%$ \\
\hline Taxanos + trastuzumab & $1,1 \%$ \\
\hline Trastuzumab & $1,1 \%$ \\
\hline $\begin{array}{l}\text { Actualmente sin esquema de de cirugía } \\
\text { quimioterapia }\end{array}$ & $5,8 \%$ \\
\hline \multicolumn{2}{|c|}{ Tratamiento hormonal } \\
\hline Radical & $38,0 \%$ \\
\hline Conservadora & $61,9 \%$ \\
\hline \multicolumn{2}{|c|}{$44,8 \%$} \\
\hline Tamoxifeno & $41,3 \%$ \\
\hline Inhibidores de la aromatasa & $3,4 \%$ \\
\hline Fulvestrant & $10,3 \%$ \\
\hline Tamoxifeno + inhibidores de la aromatasa
\end{tabular}




\section{Evaluación del dolor}

De las 93 pacientes, 53 presentaban dolor [56,9\%), de las que el $47,1 \%$ lo calificó como agudo y el $52,8 \%$ como crónico; un 82,9 \% informó de que su dolor se presentó durante o al terminar el tratamiento oncológico, y un 16,9 \% tenía dolor antes del inicio del tratamiento. De las 53 pacientes que presentaban dolor, 42 tenían antecedente de cirugía y, de estas, el 42,8\% habían sido sometidas a cirugía radical y el 52,1\% a cirugía conservadora.

De acuerdo con la escala DN4, se encontró que en 29 pacientes $(54,7 \%)$ el dolor fue clasificado como dolor nociceptivo y en 24 pacientes (45,2 \%) como dolor neuropático (Figura 2). En cuanto a la intensidad del dolor, 26 pacientes $[49,0 \%)$ reportaron dolor intenso (EVA 7-10), $23(43,4 \%$ ) dolor moderado (EVA 4-6) y solo 4 pacientes (7,5\%) presentaban dolor leve (EVA 1-3).

Las descripciones más frecuentes en cuanto a las características del dolor fueron: dolor de tipo presión [35,8 \%), ardor (47,1\%) y calor (16,9\%), y las localizaciones más frecuentes fueron el miembro superior (58,4\%), tórax [39,6\%), miembros inferiores (20,7\%) y las articulaciones [24,5\%). Un 47,1\% refirió dolor en una sola localización, un 39,6 \% en dos localizaciones y un $13,2 \%$ en más de tres localizaciones.

El manejo del dolor fue realizado con acetaminofeno en el $52,8 \%$ de las pacientes, con antinflamatorios no esteroideos (AINE) en el 5,6\%, con opioides en el $1,89 \%$ y y el $22,6 \%$ no tenían ningún tratamiento. Además, ninguna paciente estaba en tratamiento analgésico con neuromoduladores. Este manejo del dolor era automedicado en el 26,4 \% de las pacientes, y clínica de dolor solo le había formulado la medicación al 22,6\% (Tabla III).

Entre las pacientes que presentaron dolor neuropático, el 87,5\% recibieron manejo con quimioterapia y el $67 \%$ habían sido sometidas a radioterapia (Figura 3). Las pacientes con dolor neuropático se caracterizaban por haber recibido quimioterapia, manejo quirúrgico, estar en estadios IIIA, IIA y IIIB, haber recibido esquemas de quimioterapia con taxanos más antracíclicos, antracíclicos más taxanos y trastuzumab y habían recibido radioterapia. Y las pacientes que no presentaron

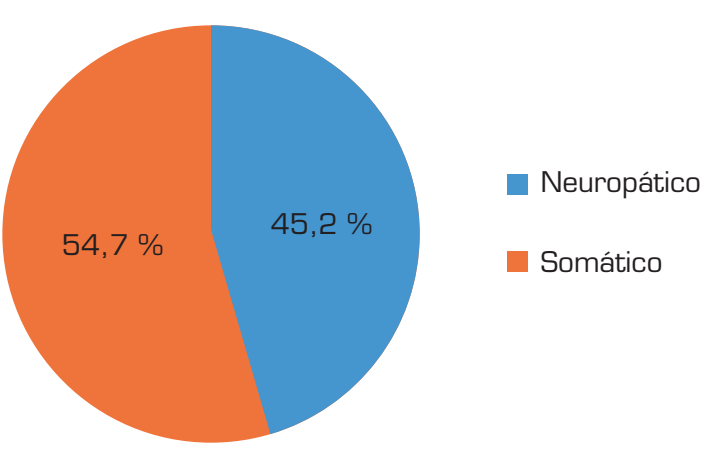

Fig. 2. Distribución del tipo de dolor. dolor neuropático se caracterizaban por no haber recibido ningún esquema de quimioterapia, estar en estadios IV y IA, estadio IIIC, IIB, no haber recibido radioterapia ni cirugía (Figura 4).

De las 26 pacientes con dolor intenso (EVA 7-10), el $19,2 \%$ no estaban recibiendo ningún tratamiento, el 38,4\% recibían manejo en monoterapia con acetaminofeno, el 7,6 \% con AINE y el 3,8\% con opioides.

\section{DISCUSIÓN}

El cáncer de mama es responsable de aproximadamente 8,2 millones de muertes anuales en el mundo (1). El tratamiento depende de la estadificación oncológica con la evaluación clínica y patológica definida por medio del sistema TNM. La piedra angular del tratamiento es el manejo quirúrgico mediante resección del tumor con márgenes oncológicos; sin embargo, múltiples estudios realizados por el Proyecto Nacional Quirúrgico de Seno e Intestino (NSABPI) demuestran

TABLA III

TRATAMIENTO ANALGÉSICO

\begin{tabular}{|c|c|}
\hline \multicolumn{2}{|l|}{ Medicación } \\
\hline Acetaminofeno & $52,8 \%$ \\
\hline Sin medicación & $22,6 \%$ \\
\hline AINE & $5,6 \%$ \\
\hline Opioides & $1,8 \%$ \\
\hline \multicolumn{2}{|l|}{ Combinación } \\
\hline Acetaminofeno + AINE & $5,6 \%$ \\
\hline Acetaminofeno +opioides & $5,6 \%$ \\
\hline AINE + opioides & $1,8 \%$ \\
\hline Acetaminofeno + AINE + opioides & $3,7 \%$ \\
\hline
\end{tabular}

AINE: antinflamatorios no esteroideos.

Esquema de quimioterapia

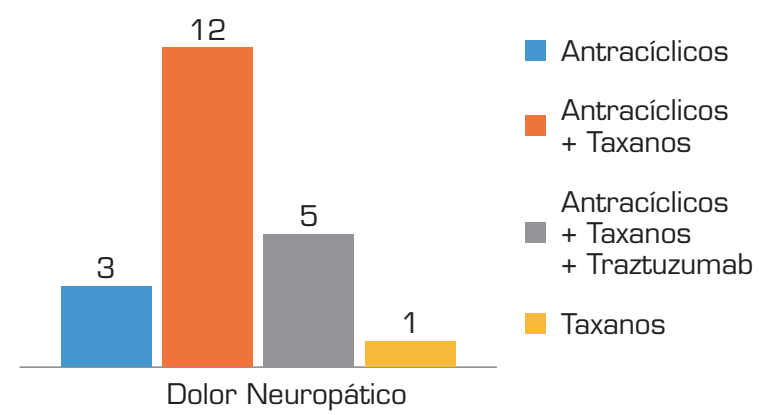

Fig. 3. Relación del tipo de tratamiento con el dolor neuropático. 


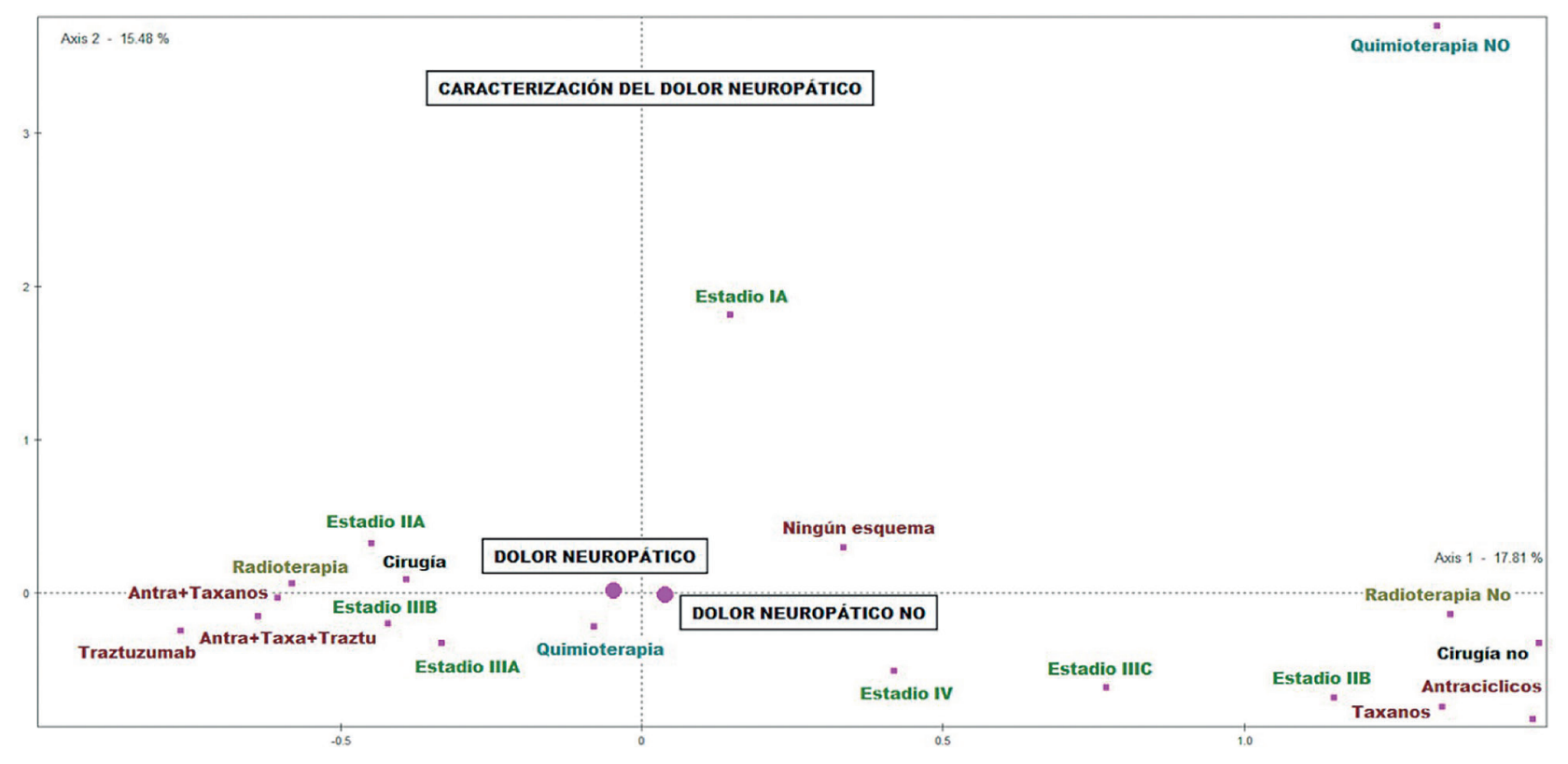

Fig. 4. Gráfica de dispersión: relación con el dolor neuropático.

un claro beneficio oncológico con la neoadyuvancia y la adyuvancia, mostrando aumento de la supervivencia y del periodo libre de enfermedad $(9,11,12)$. La IASP estima una prevalencia de dolor en el cáncer de mama del $40-89 \%$ y y $20-50 \%$ de las pacientes con cáncer de mama van a presentar dolor neuropático después de su tratamiento quirúrgico [5]. El dolor por cáncer es común, y se considera secundario a dos factores importantes: el que es producido directamente por el tumor, que provoca deformidad de estructuras adyacentes o efectos endocrinos por la secreción de sustancias inflamatorias, y el secundario al tratamiento oncológico, relacionado con las secuelas de procedimientos quirúrgicos o del tratamiento sistémico con quimioterapia y/o radioterapia $[5,8,13,14]$.

En nuestras pacientes, la prevalencia encontrada de dolor fue del 56,9 \%, hallazgo que muestra que el dolor es un síntoma frecuente y que no dista mucho de lo reportado en la bibliografía, donde encontramos prevalencias de dolor crónico de cualquier origen del $48 \%(4,10)$. De las pacientes sometidas a cirugía, el 59,1\% refirieron dolor, que podría estar relacionado con la aparición de dolor posquirúrgico persistente, el cual está reportado en la bibliografía con una prevalencia del 10-30 \% de todos los pacientes quirúrgicos, con procedimientos de alto riesgo como es la cirugía de mama, y con factores de riesgo asociados con este tipo de dolor como son mujeres jóvenes, trastornos de ansiedad, depresión, cirugías con duración mayor de 3 horas, dolor agudo postoperatorio intenso y dolor preoperatorio $(4,6,15)$. Se encontró que el $52,8 \%$ de las pacientes referían padecer dolor crónico, lo que deja en evidencia la falta de un manejo integral de estas pacientes, enfocado no solo al tratamiento en cuanto a la curación y/o control de la enfermedad, sino también al control de otras variables que pueden contribuir a la calidad de vida de las pacientes, como es el dolor $(6,9)$.
En la bibliografía encontramos reportes de neuropatía por quimioterapia del 30-40 \% $(5,13)$. En nuestras pacientes la prevalencia de dolor neuropático, medido por la escala DN4, fue del 45,2 \%, el cual puede estar en relación con la neuropatía inducida por quimioterapia, pero también por el tratamiento con radioterapia, cirugía o progresión tumoral $(8,16,17)$. A pesar de que el dolor neuropático es frecuente, no se encontró manejo analgésico con ningún neuromodulador, por lo que, siendo el dolor neuropático multifactorial y con una importante incidencia en las pacientes, se hace necesaria la creación de protocolos de manejo para identificar de manera temprana a las pacientes que tienen riesgo de presentar este tipo de dolor y, así, ante síntomas y signos tempranos instaurar un manejo adecuado, además de realizar investigaciones con moléculas que tengan relevancia en la prevención de este tipo de dolor.

Como parte del tratamiento adyuvante del cáncer de mama se utiliza la hormonoterapia, que por lo general se usa por periodos de 5 años. Estas sustancias químicas pueden generar dolor per se. En nuestro estudio se evidencia que de las pacientes que recibieron hormonoterapia, casi la mitad recibían tamoxifeno y la otra mitad inhibidores de la aromatasa; estos últimos pueden provocar como efectos secundarios artralgias, las cuales pueden aparecer en cualquier momento del tratamiento. En este estudio se encontró la presencia de dolores articulares en un 24,5 \% (18). Respecto al tratamiento analgésico, se encontró que la mayoría de las pacientes [52,8 \%) recibieron acetaminofeno, y un 22,6 \% no recibieron tratamiento, lo que deja en evidencia que, a pesar de que las pacientes refieren dolor de intensidad moderada a intensa, el control del dolor es pobre, dado que en las recomendaciones de la escalera analgésica de la Organización Mundial de la Salud el acetaminofeno forma parte del primer escalón 
para un uso en dolores de leve intensidad, y para el dolor de intensidad moderada a intensa se recomiendan fármacos del segundo y tercer escalón, con uso de opioides débiles o fuertes. Por otro lado, hay un importante porcentaje de pacientes que no recibieron tratamiento analgésico, lo que demuestra la centralización en el manejo oncológico dejando de lado síntomas como el dolor, que sin embargo tiene un gran impacto en la calidad de vida de las pacientes.

Se pone de manifiesto la falta de calidad asistencial en el manejo del dolor de estas pacientes mediante un tratamiento rehabilitador e intervencionista (técnicas de bloqueo nervioso, articular o interfascial) perioperatorio o posterior al tratamiento quirúrgico].

Nuestros resultados permiten conocer la dimensión del dolor en pacientes con cáncer de seno de la unidad de oncología de un hospital de cuarto nivel, y podrían contribuir al planteamiento de medidas específicas para su abordaje y a la creación de políticas institucionales que garanticen un abordaje integral del paciente.

\section{AGRADECIMIENTOS}

A todas las pacientes participantes en este estudio y al Servicio de Oncología del Hospital San José por permitir la recolección de datos.

\section{CONFLICTO DE INTERESES}

Artículo de investigación presentado como requisito para optar al título de Especialista en Dolor y Cuidado Paliativo de la Fundación Universitaria de Ciencias de la Salud.

Los autores declaramos no presentar conflicto de interés.

\section{FINANCIACIÓN DEL PROYECTO}

Esta investigación no recibió apoyo financiero institucional.

\section{BIBLIOGRAFÍA}

1. WHO. Breast Cancer Estimated Incidence, Mortality and Prevalence Worldwide in 2012 [internet]. 2012 Disponible en: http://globocan.iarc.fr/Pages/fact_sheets_cancer.aspx.

2. Instituto Nacional de Cancerología ESE. Guía de práctica clínica para la detección temprana, tratamiento integral, seguimiento y rehabilitación del cáncer de mama. Bogotá: Ministerio de Salud y Protección Social - Departamento
Administrativo de Ciencia Tecnología e Innovación en Salud (COLCIENCIAS); 2013.

3. American Cancer Society. Tasas de supervivencia del cáncer de seno 2017. Disponible en: https://www.cancer.org/es/ cancer/cancer-de-seno/comprension-de-un-diagnostico-de-cancer-de-seno/tasas-de-supervivencia-del-cancer-de-seno.html.

4. Farquhar-Smith P, Brown M. Persistent Pain in cancer survivors: pathogenesis and treatment options. Pain: Clinical Updates. 2016;24(4):1-8. Disponible en: https:// s3.amazonaws.com/rdcms-iasp/files/production/public/ AM/Images/PCU/PCU\%2024-4_web.pdf.

5. Satija A, Ahmed SM, Gupta R, Ahmed A, Rana SP, Singh SP, et al. Breast cancer pain management - A review of current \& novel therapies. Indian J Med Res. 2014;139(2):216-25.

6. Rosenquist E. Evaluation of chronic pain in adults. Up to date; last update: Oct 04, 2018. Disponible en: https://www. uptodate.com/contents/evaluation-of-chronic-pain-in-adults

7. José Cid C, Juan Pablo Acuña B, Javier de Andrés A, Luis Díaz J, Leticia Gómez-Caro A. ¿Qué y cómo evaluar al paciente con dolor crónico? Evaluación del paciente con dolor crónico. Revista Médica Clínica Las Condes. 2014;25(4):687-97.

8. Miltenburg NC, Boogerd W. Chemotherapy-induced neuropathy: A comprehensive survey. Cancer Treat Rev. 2014;40(7):872-82.

9. Teshome M, Hunt KK. Neoadjuvant therapy in the treatment of breast cancer. Surg Oncol Clin N Am. 2014;23(3):505-23.

10. Díaz R, Marulanda F. Dolor crónico nociceptivo y neuropático en población adulta de Manizales (Colombia). Acta Medica Colombiana. 2011;36:10-7.

11. Esserman M. Clinical features, diagnosis, and staging of newly diagnosed breast cancer 2017 Disponible en: http:// www.uptodate.com

12. Esserman LJ, Shieh Y, Rutgers EJ, Knauer M, Retèl VP, Mook $S$, et al. Impact of mammographic screening on the detection of good and poor prognosis breast cancers. Breast Cancer Res Treat. 2011;130(3):725-34.

13. Park HJ. Chemotherapy induced peripheral neuropathic pain. Korean J Anesthesiol. 2014;67(1):4-7.

14. Loprinzi CL. Prevention and treatment of chemotherapyinduced peripheral neuropathy 2017. Up to date; last update: Mar 28, 2019. Disponible en: https://www.uptodate. com/contents/prevention-and-treatment-of-chemotherapyinduced-peripheral-neuropathy

15. Feizerfan $A$, Sheh $G$. Transition from acute to chronic pain. CEACCP. 2015;15(2):98-102.

16. Velasco R, Bruna J. Chemotherapy-induced peripheral neuropathy: an unresolved issue. Neurologia. 2010;25(2):116-31.

17. Han Y, Smith MT. Pathobiology of cancer chemotherapyinduced peripheral neuropathy (CIPN). Front Pharmacol. 2013;4:156.

18. Caicedo JJ, Quintero E, Robledo JF, Perry F, Ramírez C, Duarte C, et al. Cáncer de seno y hormonoterapia. Estado actual. Rev Colomb Cir; 2007;22(1):47-71. 